\title{
A variant near DHCR24 associates with microstructural properties of white matter and peripheral lipid metabolism in adolescents
}

Short title: DHCR24 links brain-tissue microstructure and peripheral metabolism

Eeva Sliz ${ }^{1,2,3}$, Jean Shin ${ }^{1,2}$, Catriona Syme ${ }^{1,2}$, Yash Patel ${ }^{4}$, Nadine Parker ${ }^{4}$, Louis Richer ${ }^{5}$, Daniel Gaudet ${ }^{6}$, Steffany Bennett ${ }^{7}$, Tomas Paus ${ }^{4,8}$, Zdenka Pausova ${ }^{1,2^{*}}$

1. The Hospital for Sick Children, University of Toronto, Toronto, ON, Canada

2. Departments of Physiology and Nutritional Sciences, University of Toronto, Toronto, ON, Canada

3. Center for Life-Course Health Research and Computational Medicine, Faculty of Medicine, University of Oulu, and Biocenter Oulu, Oulu, Finland

4. Bloorview Research Institute, Holland Bloorview Kids Rehabilitation Hospital, Toronto, ON, Canada

5. Department of Health Sciences, Université du Québec à Chicoutimi, Chicoutimi, QC, Canada

6. Clinical lipidology and rare lipid disorders Unit, Community Genetic Medicine Center, Department of Medicine, Université de Montréal, and ECOGENE-21, Chicoutimi, QC, Canada

7. Neural Regeneration Laboratory, Ottawa Institute of Systems Biology, University of Ottawa, Ottawa, Canada

8. Departments of Psychology and Psychiatry, University of Toronto, Toronto, ON, Canada

* Corresponding author:

Zdenka Pausova, MD, FAHA

Senior Scientist, Hospital for Sick Children

Professor, Departments of Physiology and Nutritional Sciences

University of Toronto

Peter Gilgan Centre for Research and Learning

686 Bay Street, Rm. 10-9705

Toronto, ON M5G 0A4

Canada

Phone: (416) 813-7654/304340

Fax: (416) 813-5771

E-mail: zdenka.pausova@sickkids.ca

Figures: 5

Tables: 2

Supplementary figures: 9

Supplementary tables: 3

Number of references: 62 
42

43

44

45

46

47

48

49

50

51

52

53

54

55

56

57

58

59

\begin{abstract}
Visceral adiposity has been associated with altered microstructural properties of white matter in adolescents. Previous evidence suggests that circulating phospholipid PC(16:0/2:0) may mediate this association. To investigate the underlying biology, we performed a genome-wide association study (GWAS) of the shared variance of visceral fat, $\mathrm{PC}(16: 0 / 2: 0)$, and white matter microstructure in 931 adolescents from the Saguenay Youth Study. We further studied the metabolomic profile of the GWAS-lead variant. Visceral fat and white matter microstructure were assessed with magnetic resonance imaging. Circulating metabolites were quantified with serum lipidomics and metabolomics. We identified a genome-wide significant association near DHCR24 (Seladin-1) encoding a cholesterol-synthesizing enzyme (rs588709, $\left.p=3.6 \times 10^{-8}\right)$; rs588709 was also associated nominally with each of the three traits (white matter microstructure: $p=2.1 \times 10^{-6}$, $\mathrm{PC}(16: 0 / 2: 0): p=0.005$, visceral fat: $p=0.010)$. We found that the metabolic profile associated with $\mathrm{rs} 588709$ resembled that of a TM6SF2 variant impacting very-low-density lipoprotein (VLDL) secretion and was only partially similar to that of a HMGCR variant. This suggests that the effect of rs588709 on VLDL lipids may arise due to altered phospholipid rather than cholesterol metabolism. The rs588709 was also nominally associated with circulating concentrations of omega- 3 fatty acids in interaction with visceral fat and $\mathrm{PC}(16: 0 / 2: 0)$, and these fatty acid measures showed robust associations with white matter microstructure. Overall, the present study provides evidence that the DHCR24 locus may link peripheral metabolism to brain microstructure, an association with implications for cognitive impairment.
\end{abstract}


60

61

62

63

64

65

66

67

68

69

70

71

72

73

74

75

76

77

78

79

80

81

82

83

84

85

86

87

88

89

90

91

92

93

94

95

96

97

98

99

100

101

102

103

104

105

106

107

108

109

110

111

112

113

\section{INTRODUCTION}

Previous evidence advocates that obesity-related changes in the peripheral biology, such as 'low-grade' systemic inflammation, may have adverse effects on brain health $[1,2]$. Visceral accumulation of body fat may be especially deleterious: we and others have shown that visceral fat (VF), quantified with magnetic resonance or computer tomography imaging, associates with alterations in microstructural properties of white matter (WM) [3], higher incidence of WM lesions [4], lower brain volume [5], and lower cognitive functioning [6].

We have previously identified a novel circulating phospholipid PC(16:0/2:0) [7], which correlates closely with systemic inflammation, and links visceral adiposity to altered WM microstructure and lower cognitive functioning [8]. Circulating phospholipids can function as modulators of inflammation and oxidative stress [9]. They may influence the integrity of endothelial barriers, including the blood-brain barrier [10, 11], and thus impact the transition of obesity-related systemic inflammation to neuroinflammation [12]. In turn, neuroinflammation may lead to subtle alterations in WM microstructure [13] that are detectable with magnetic resonance imaging (MRI) and that may contribute to cognitive impairment.

Circulating phospholipids are generated by enzymatic remodeling of biological membranes, such as the phospholipid bilayers of cellular membranes, or the phospholipid monolayers of lipoprotein shells [9, 14]. The main sources of circulating phospholipids are the membranes of blood and endothelial cells, and of circulating lipoproteins. Phospholipid membrane remodeling may be modulated by systemic inflammation, such as that induced by obesity $[8,9,15]$. Phospholipid membrane remodeling also occurs in other tissues, including the brain, where it may be modulated by neuroinflammation [12]. Systemic inflammation and neuroinflammation, as well as alterations in circulating and brain-tissue phospholipids have been linked to cognition and dementia [2, 16-18].

To add understanding to the underlying biology linking visceral adiposity, circulating phospholipid PC(16:0/2:0) and WM microstructure, we performed a genome-wide association study (GWAS) of the variance shared by these three variables. We identified a significant locus near the gene encoding 24dehydrocholesterol reductase (DHCR24), which is an enzyme converting desmosterol to cholesterol [19]. In the brain, DHCR24 has been proposed to play a neuroprotective role by reducing oxidative stress and apoptosis [20-24]. In mice, Dhcr24 deficiency decreases cholesterol levels in cellular phospholipid membranes and alters the composition and function of lipid rafts critical for cell signalling [20]. In the present study, we show that the GWAS-lead variant near DHCR24 is associated with circulating levels of very-low-density lipoproteins (VLDL) that require, for their synthesis, the formation of phospholipid monolayer incorporating cholesterol.

\section{METHODS}

\section{Study population}

The Saguenay Youth Study (SYS) is a population-based study conducted in the Saguenay-Lac-St.-Jean region of Quebec, Canada, which is aimed at investigating the etiology and early stages of common cardiometabolic and brain diseases [25]. The SYS includes 486 families with a total of 1,028 adolescents and 962 parents [25]. We performed a GWAS of shared variance between visceral adiposity, inflammation, and WM microstructure in a subset of 872 adolescents in whom complete genotype information, targeted lipidomics data [7], and brain and abdominal MRI imaging were available. We further studied the metabolomic profile of the GWAS-lead variant in 931 adolescents. Written assent was obtained from all participants, and the study was approved by the research ethics committees of Chicoutimi Hospital (Chicoutimi, QC, Canada) and the Hospital for Sick Children (Toronto, ON, Canada).

\section{Study genotype and phenotype quantifications}

A detailed description of the study genotype and phenotype assessments is given in Supplementary Methods. In short, genotyping was completed using Human610-Quad and HumanOmniExpress BeadChips (Illumina). Subsequent genotype imputations were performed using 1000 Genome CEU reference panel. MRI was conducted using a Gyroscan NT 1.0-T scanner (Philips Healthcare). WM microstructure was assessed as normalized WM T1-weighted signal intensity (T1W-SI), and quantity of VF was determined from T1W images of the abdomen. Quantification of the circulating phospholipid PC(16:0/2:0) was performed using 
nanobore liquid chromatography-nano electrospray ionization-tandem tandem mass spectrometry (NLCNESI-MS/MS) [7]. Quantification of a total of 228 circulating metabolic traits was performed with a nuclear magnetic resonance (NMR) metabolomics platform (Nightingale Health Ltd) [26]. All circulating measures were quantified from fasting serum samples.

\section{Statistical analysis}

The aim of this study was to search for genetic loci explaining the shared variance of VF, PC(16:0/2:0) and WM microstructure. We further determined the metabolomic effects of the GWAS-lead variant and explored its genome biology in open-access data.

\section{Principal component analysis}

To obtain a numerical estimate for the shared variance between $\mathrm{VF}$, circulating concentration of $\mathrm{PC}(16: 0 / 2: 0)$, and WM microstructure, we performed principal component analysis (PCA) of the three variables. Prior to this analysis, VF and PC(16:0/2:0) were log-transformed ensure normality, and each variable was then adjusted for age and sex (and height for VF). Using the correlation matrix of the adjusted variables, we carried out PCA and obtained the $1^{\text {st }}$ principal component $(\mathrm{PC} 1)$. The PC1 was quantilenormalized using a rank-based inverse normal transformation and used as the study phenotype in a subsequent GWAS.

\section{Genome-wide association}

The GWAS of PC1 was conducted in 872 SYS adolescents, using a mixed effects model-based method implemented in ProbABEL [27], which enables adjusting for family relatedness.

We further tested the GWAS-lead variant for associations with each VF, PC(16:0/2:0) and WM microstructure individually. The variables were adjusted for age, sex, (and height for VF) and common family environment using the 'Imekin' function from the 'coxme' R package, and subsequently inverse ranktransformed to normality. Linear models were fitted where the adjusted and transformed phenotypes served as outcomes and the genotype of the GWAS-lead variant encoded as 0,1 , and 2 (representing the number of GWAS-effect alleles) as the explanatory variable.

\section{GWAS-lead variant and circulating metabolic traits}

Linear regression models were fitted to evaluate associations of the GWAS-lead variant with 228 circulating metabolic traits. Here, each of the metabolic traits was an outcome and the genotype was the explanatory variable. Prior to these analyses, each metabolic trait was adjusted for age, sex, and common family environment using 'Imekin' from 'coxme' R package, as described above, and inverse rank-transformed to normality. We further determined the associations of the GWAS-lead variant with circulating metabolic traits in interaction with VF and PC(16:0/2:0) using the same method.

Due to the correlation of the metabolic traits, the number of independent tests performed is lower than the total number of metabolic traits studied. The number of principal components explaining $95 \%$ of the variation in the SYS adolescents metabolomics data was 29 and, thus, we considered statistical significance at $p<0.002(0.05 / 29)$ accounting for 29 independent tests [28].

\section{Corroboration of results within random splits}

To provide additional statistical support for the identified metabolomic associations with the GWAS-lead variant, we used an approach described by Sabourin et al. [29]. For this, the SYS adolescent data were randomly split into two halves, and the linear models described above were fitted to each of the halves. We repeated the splitting and fitting steps 1,000 times and calculated the corroboration score: the proportion of random splits for which 1) the association between DHCR24 genotype and metabolic measure was significant $(p<0.05)$ in both halves; and 2$)$ the effect estimates did not statistically differ between the halves. High corroboration scores (i.e., $>0.4$ ), suggest that identified associations are more likely to be replicated in an independent sample [29].

Except for the GWAS, all above analyses were performed in R version 3.3.1. 


\section{Open-access data look-ups}

The impact of the GWAS-lead variant on expression of neighbouring genes was studied in Genotype-Tissue Expression (GTEx) [30]. Brain expression of these genes was studied in Brain eQTL Almanac (Braineac) (www.braineac.org) [31]. To evaluate if the expression of these genes varies with age and to study their coexpression with other genes, further tests were performed using expression data of cortical brain samples from a total of 572 individuals acquired from five human post-mortem databases (Allen Human Brain Atlas [32, 33], BrainCloud [34, 35], Braineac [31], BrainSpan [36], and GTEx [30]). Using a similar technique as Parker et al. [37], the expression of each gene was scaled within each sampled region and grouped into the four lobes of the brain. To test lifespan variation in expression, donor age was regressed on lobar expression adjusting for sex and repeated donor samples per lobe (random effect for donor ID). Co-expression of the genes near the GWAS-lead variant was tested separately with all other 16,036 genes measured in each of the five gene expression databases. Linear mixed models were used to adjust for age, sex, and repeated measures as random effect. For each of the tested genes, the top $1 \%$ of positively co-expressed genes (as determined by smallest p-values of the gene effects term) were then tested for enrichment with neural cell specific markers derived from PsychENCODE (adult) [38] and single-cell RNAseq data by Lake et al. [39]. The same sets of co-expressed genes were used in gene ontology (GO) enrichment analysis conducted using ToppGene tool [40], limiting GO groups with a maximum of 250 genes.

The associations of the GWAS-lead variant with DNA methylation were studied in DNA methylation quantitative trait loci database (mQTLdb) [41]. In addition, the annotation of the GWAS-lead variant was explored using HaploReg v.4.1 [42].

To estimate possible biological origins of the metabolic associations of the GWAS-lead variant, we compared metabolic effects of this variant with the metabolic effects of two other gene variants with known molecular function. These were 3-hydroxy-3-methylglutaryl-CoA reductase (HMGCR) rs12916-T and transmembrane 6 superfamily member 2 (TM6SF2) rs58542926-T. HMGCR is a key enzyme in cholesterol synthesis [43], and TM6SF2 is involved in the regulation of hepatic secretion of VLDL [44]. The two variants inhibit the normal function of the two genes [44, 45]. We extracted the metabolomic effects of these variants from a metabolomics GWAS performed in 24,925 individuals of European ancestry [28]. The metabolomics platform used by Kettunen et al. is an older quantification version of the platform used in the present study and it covers 123 metabolic measures. The overall similarities between the metabolic effect estimates of the GWAS-lead variant and TM6SF2 rs58542926-T or HMGCR rs12916-T were estimated using the linear fit of effect estimates on 111 metabolic measures available in both studies. To overcome the differences in the effect sizes of the absolute effects, we scaled the metabolic effects to a common factor that was in this case the lowering effect on apolipoprotein B (apoB) concentration. This method is advantageous when studying metabolic pathways of interest rather than magnitudes of effects [46]. To replicate the present findings, the effects of the GWAS-lead variant were acquired from the same metabolomics GWAS [28].

\section{RESULTS}

The study sample included up to 931 adolescents from SYS (Table 1). The PC1 of VF, circulating concentration of PC(16:0/2:0), and WM microstructure (T1W-SI) explained 51.1\% of the shared variance among the three variables. The respective factor loadings were $0.73,-0.71$, and 0.70 , reflecting the originally described relationships between the three variables, i.e., individuals with more VF and higher WM T1W-SI tended to have lower circulating level of PC(16:0/2:0) [8].

\section{Genome-wide significant association near DHCR24}

The GWAS of PC1 identified a genome-wide significant locus in $1 \mathrm{p} 32.3$ (rs588709; $p=3.58 \times 10^{-8}$; Figure 1). When studied individually, the GWAS-lead variant was also nominally associated with each of the three variables in the SYS adolescents (WM T1W-SI: $p=2.1 \times 10^{-6} ; \mathrm{PC}(16: 0 / 2: 0): p=0.005$; VF: $p=0.010$; Figure $\mathrm{S} 1)$. The rs588709 is part of a $\sim 41 \mathrm{~kb}$ linkage disequilibrium block overlapping with the 3 '-untranslated region of DHCR24 (Figure 1). The other genes in this locus are lymphocyte expansion molecule (LEXM), tetratricopeptide repeat domain 22 (TTC22) and an antisense gene RP11-67L3.4. The allele frequency of the GWAS effect-allele rs588709-G was 0.36 in the SYS population, which was slightly lower than the global frequency (0.44) in the 1000 Genomes Phase 3 data [47]. 
The GWAS-lead variant rs588709 associates with altered gene expression and DNA methylation, and changes NRSF/REST-binding sequence

Based on GTEx [30], the rs588709 genotype is associated with altered mRNA expression of DHCR24 as well as LEXM, TTC22 and RP11-67L3.4 (Figures S2-S5). DHCR24 is expressed in multiple tissues, with the highest levels seen in the adrenal gland, spinal cord, esophagus, and liver (Figure S2 B). LEXM and TTC22 are expressed primarily in the esophagus, and RP11-67L3.4 in the testes (Figures S3-S5 B). DHCR24, TTC22, and LEXM are also expressed in the brain, with DHCR24 demonstrating the highest levels (Figures S2-S4 C).

The results obtained using human cerebrocortical gene expression data from open-access sources suggest that DHCR24 expression increases in the frontal and temporal lobe with a marginal increase in the occipital lobe during the first two decades of life (Figure 2). Lobar expression of TTC22 and LEXM does not vary with age (Figure S6).

Using the same human cerebrocortical gene expression data, we found that the top $1 \%$ of genes positively co-expressed with DHCR24 is enriched for oligodendrocyte- and excitatory neuron-specific markers (Bonferroni-adjusted $p$-values $8.05 \times 10^{-6}$ and $8.10 \times 10^{-8}$, respectively), as well as the myelin-sheath cellular compartment (Figure S7). Contrastingly, genes co-expressed with TTC22 and LEXM were not found to be enriched with any neural cell-specific markers (Figure S7). GO analysis of genes co-expressed with TTC22 suggests enrichment of membrane-related cellular compartments, such as brush-border membrane and sarcoplasmic reticulum.

Data from mQTLdb [41] indicated that rs588709 associates with DNA methylation in blood cells at four CpG sites during pregnancy, two CpG sites at birth, five $\mathrm{CpG}$ sites in childhood, six CpG sites in adolescence, and one $\mathrm{CpG}$ site in middle-age (Table $\mathrm{S} 1$ ). These $\mathrm{CpG}$ sites were located in the regulatory regions of DHCR24, LEXM and TTC22 (Figure S8). The GWAS-effect allele G was associated with higher DNA methylation at all the listed $\mathrm{CpG}$ sites. The $\mathrm{CpG}$ located in the proximal promoter of DHCR24, cg27168858, was differentially methylated in association with rs588709 in childhood and adolescence but not in adulthood (Table S1 and Figure S8).

Based on the HaploReg [42] analysis, the rs588709 alters a regulatory motif of five transcription factors (Table S2). These include Neuron-Restrictive Silencer Factor (NRSF) that is also known as RE1-Silencing Transcription Factor (REST). NRSF/REST functions as a repressor of neuronal genes in non-neuronal tissues [48]. Its active stage correlates with cognitive preservation and longevity, while lower activity has been linked with neurodegeneration and Alzheimer's disease [49].

DHCR24 rs588709 associates with circulating levels of very-low-density lipoprotein particles and lipids DHCR24 rs588709-G was associated with lower circulating level of apoB-containing lipoprotein particles (Figure 2). The associations were the most robust for the triglyceride-rich VLDL particle subfractions: rs588709-G was associated with lower particle concentrations of the five largest (XXL-S) VLDL subfractions (respective $p$-values of $0.00028,0.00017,0.00023,0.00074$ and 0.0014 ) and lower total lipids within these subfractions ( $p$-values of $0.00030,0.00019,0.00023,0.00072$, and 0.0016 ). The rs $588709-G$ was associated also with lower triglycerides in all the 6 VLDL subfractions, lower phospholipids in XXL-M VLDL subfractions, cholesteryl esters in XXL-L VLDL subfractions, and lower free cholesterol in XXL-S VLDL subfractions. Consequent reductions were observed in total serum triglycerides $(p=0.00048)$ and VLDL triglycerides $(p=0.00051)$ (Figure 3$)$. From the non-lipid measures, the rs588709-G showed the strongest effects on amino acids leucine $(p=0.0015)$ and glycine $(p=0.0013)$ (Figure 4$)$, and a nominally significant effect ( $p=0.0081$ ) on glycoprotein acetylation (GlycA), a marker of systemic inflammation [50]. The effect estimates for all the 228 metabolic associations in SYS adolescents are plotted in Figure S9 and tabulated in Table S3 together with the corroboration scores. The associations of rs588709 with VLDL lipids showed high corroboration rates (e.g., >0.4), suggesting that the associations are less likely to be an artefact of our analysed sample and hence more likely to be replicated in an independent sample [29]. 
To evaluate the potential molecular mechanisms, we compared the rs588709-G association profile with the association profiles of two genotypes with known molecular functions. The variant rs12916-T downregulates expression of HMGCR [45], encoding a key enzyme in cholesterol synthesis. The variant rs58542926-T is a loss-of-function allele diminishing the normal function of TM6SF2 in VLDL particle secretion [51, 52]. DHCR24 rs588709-G showed a matching association profile with the TM6SF2 rs58542926-T (Figure 5, $R^{2}=0.74$ ). The metabolic effect estimates of the DHCR24 and TM6SF2 variants were concordant throughout the lipoprotein subfraction particle concentrations and the lipid content of these subfractions (Figure S10). The effects of the DHCR24 variant were only somewhat similar to the HMGCR variant (Figure 5, $R^{2}=0.41$ ): HMGCR rs12916-T showed an association pattern where the lowering effect on the apoB-containing particles gets more robust when the particle size decreases, while this is not the case for DHCR24 rs588709G nor TM6SF2 rs58542926-T (Figure S10).

We further tested if the GWAS-lead variant is associated with circulating metabolic traits in interaction with $\mathrm{VF}$ and PC(16:0/2:0). The 3-way interaction term of DHCR24 rs588709, VF and PC(16:0/2:0) showed nominally significant $(p<0.05)$ associations with circulating levels of $\beta$-hydroxybutyrate, docosahexaenoic acid (DHA), DHA to total fatty acids ratio (DHA\%), omega-3 fatty acids to total fatty acids ratio (n-3FA\%) and lactate (Table S4). DHA, DHA\% and n-3FA\% were also significantly associated with WM microstructure (respective p-values $7.79 \times 10^{-12}, 6.81 \times 10^{-7}$, and $2.94 \times 10^{-7}$ ).

\section{DISCUSSION}

We identified a genetic locus associated with the shared variance of visceral adiposity, serum levels of phospholipid PC(16:0/2:0), and WM microstructure in adolescents. The locus is near DHCR24 encoding an enzyme that converts desmosterol to cholesterol. Previous studies have shown that DHCR24 plays a role in brain biology. We show that DHCR24 expression in the brain increases during childhood and adolescence, suggesting DHCR24 may be important for brain maturation. We also present here that the co-expression network of DHCR24 in the brain is enriched for neural cell-specific markers, linking DHCR24 to brain biology.

DHCR24, also known as selective Alzheimer's disease indicator-1 (Seladin-1), was originally identified as being down-regulated in the brains of patients with Alzheimer's disease [21]. Later on, the concept of DHCR24 being a selective indicator of Alzheimer's disease was questioned, as the mRNA-expression studies provided inconsistent results (summarized by Sharpe et al., [53]). Nonetheless, much of the evidence speaks for DHCR24 being neuroprotective, having anti-inflammatory, anti-oxidative and anti-apoptotic effects [20, 21, 24]. DHCR24 deficiency decreases cholesterol levels in cell membranes compromising the formation and stability of lipid rafts $[20,21]$, which may decrease glutamate uptake and thus increase the potential for neuronal excitotoxicity [54]. Neuronal excitotoxicity, in turn, may promote accumulation of A $\beta$ peptide and thus augment the risk for neurodegeneration and Alzheimer's disease [20]. Finally, loss-offunction mutations of DHCR24 cause desmosterolosis [55], which characterized by accumulation of desmosterol in the brain resulting in multiple congenital anomalies, including microcephaly and near agenesis of corpus callosum [56].

In addition to the shared variance of visceral adiposity, serum levels of phospholipid PC(16:0/2:0), and WM microstructure, we found that the DHCR24 locus is associated with peripheral lipoprotein metabolism in adolescents. Recognizing the cholesterol-synthesizing activity of DHCR24, we speculated that the VLDL lipid-lowering effect of DHCR24 rs588709-G could be due to deficiency in cellular cholesterol. Cholesterol is incorporated in small amounts into lipoprotein phospholipid-monolayers, and endogenous cholesterol synthesis has been associated with VLDL secretion rate [57]. Unexpectedly, we found that the metabolic association profile of the DHCR24 rs588709-G was only partially similar to the profile of HMCGR rs12916$\mathrm{T}\left(R^{2}=0.41\right)$, which is known to inhibit cholesterol synthesis by downregulating expression of HMG-CoA reductase, the principal rate-limiting enzyme of cholesterol synthesis [45]. Compared with the effects of DHCR24 rs588709-G, HMGCR rs12916-T showed a greater lowering effect on the intermediate and lowdensity lipoprotein (IDL, LDL) particle subfractions (Figure S6). The greater lowering effect on IDL and LDL particles arises from the upregulated expression of LDL receptor and enhanced hepatic uptake of IDL and LDL particles that occurs in parallel with the decrease in the cellular cholesterol concentration [58]. 
These observations suggest that the lowering effect on VLDL lipids associated with DHCR24 rs588709-G is likely to be due to a mechanism other than a reduction in cellular cholesterol.

We found that the metabolic association profile of DHCR24 rs588709-G was similar to the metabolic association profile of TM6SF2 rs58542926-T. This loss-of-function allele impairs VLDL secretion due to shortage in phosphatidylcholines resulting in hepatic triglyceride accumulation [52] and concordant reduction in circulating lipid levels [46]. Based on the highly matching metabolic profiles of the two variants $\left(R^{2}=0.74\right)$ and the fact that phosphatidylcholines are the only phospholipids required for the assembly and secretion of VLDL particles [59], it is possible that the VLDL-lowering effect of the DHCR24 rs588709-G could arise from altered phosphatidylcholine metabolism. Supportive of this possibility, in the present study, DHCR24 rs588709-G was nominally associated with lower circulating level of phosphatidylcholine $\mathrm{PC}(16: 0 / 2: 0)$.

Our observation that the interaction term of DHCR24 rs588709, VF and PC(16:0/2:0) was nominally associated with circulating levels of $\beta$-hydroxybutyrate, DHA, DHA $\%$ and n-3FA\% suggests that the DHCR24 locus may influence fatty acid metabolism in interaction with VF and PC(16:0/2:0). DHA, DHA\% and $\mathrm{n}-3 \mathrm{FA} \%$ levels were also robustly associated with white matter microstructure. DHA is a major polyunsaturated fatty acid in the central nervous system, where it is used for the biogenesis and maintenance of neuronal membranes [60]. It has been shown that DHA or high fat exposures decrease DHCR24 expression in human studies [61, 62], providing supportive evidence for a possible molecular link between DHCR24 and fatty acid metabolism.

To the best of our knowledge, DNA sequence variants in or near DHCR24 have not previously been associated with metabolic or adiposity traits in human studies. Epigenome-wide studies, however, have identified DNA methylation sites annotated to DHCR24 to be differentially methylated in association with circulating triglycerides and HDL cholesterol levels [63], and with waist circumference [64]. Using data from mQTLdb [41], we found that DHCR24 rs588709 is associated with differential methylation at a CpG in DHCR24 during childhood and adolescence but not during adulthood (Table S1), suggesting that DHCR24 rs588709 may associate with differential activity of DHCR24 during childhood/adolescence versus adulthood. Our observation that DHCR24 expression in frontal and temporal lobes increases during childhood and adolescence (Figure S5) supports this possibility. According to previous studies, the demand for cholesterol by the brain is highest during development, including the brain maturation during childhood and adolescence [65], when it is required for myelination, among others [66]. Further, Dhcr24 expression is down-regulated during aging in wild-type mice [67]. In keeping with the aforementioned, we observed that the same variant is not associated with any of the studied phenotypes in SYS parents $(\mathrm{N}=527$; data available on request). The rs588709 variant also shows null metabolic effects in the metabolomics GWAS of European adult populations [28].

The use of population isolates, such as the French-Canadians from Saguenay, enhances the power to identify genetic loci in GWAS settings [68]. Given that the present results are from a single population, the replication of the findings in other population samples would be of high value. Also, we must acknowledge that rs588709 may not be the causal variant, but it may tag some other, possibly non-genotyped sequence variant, that could influence the studied traits. Further, we cannot exclude the possibility that DHCR24 is not the underlying gene, but the associations may be mediated by altered function of a different gene within the identified locus; the rs588709 variant is associated with expression level of two other nearby genes, as well as DNA methylation at the regulatory sequences of these genes. However, when considering the genomic evidence, the other genes seem less likely involved. Their expression in the brain is not regulated developmentally and is lower than that of DHCR24. Further, they are not expressed in the liver, which makes them less likely candidates for the metabolomic effects. Finally, the relevance of DHCR24 to brain biology is highlighted by the fact that the top $1 \%$ of genes co-expressed with DHCR24 is enriched for neural cellspecific markers, while this is not the case for the other genes.

The present results show that DHCR24 rs588709-G is associated with favourable brain and peripheral health properties in the SYS adolescents. The metabolic association profile of the rs588709-G suggests a novel role for DHCR24 in triglyceride-rich lipoprotein metabolism. Overall, the present results provide genetic 
384 evidence supporting the hypothesis that a shared biological mechanism, possibly involving omega-3 fatty 385 acids, and DHA in particular, could influence peripheral metabolism and brain health during adolescence. 


\section{Acknowledgements}

The authors thank the following individuals for their contributions in data acquisition and storage in the SYS: Manon Bernard (database architect, The Hospital for Sick Children), Hélène Simard and her team of research assistants (Cégep de Jonquière), and Jacynthe Tremblay and her team of research nurses (Chicoutimi Hospital). The authors thank all participants who took part in the Saguenay Youth Study. The Genotype-Tissue Expression (GTEx) Project was supported by the Common Fund of the Office of the Director of the National Institutes of Health, and by NCI, NHGRI, NHLBI, NIDA, NIMH, and NINDS. The data used for the analyses described in this manuscript were obtained from the GTEx Portal on 04/01/2019 and dbGaP accession number phs000424.v7.p2 on 04/01/2019.

\section{Conflict of Interest}

Authors declare that there are no competing financial interests in relation to the present work. 


\section{References}

1. Pou KM, Massaro JM, Hoffmann U, Vasan RS, Maurovich-Horvat P, Larson MG, et al. Visceral and subcutaneous adipose tissue volumes are cross-sectionally related to markers of inflammation and oxidative stress: The Framingham Heart Study. Circulation. 2007; 116:1234-1241.

2. Spyridaki EC, Avgoustinaki PD, Margioris AN. Obesity, inflammation and cognition. Curr Opin Behav Sci. 2016;9:169-175.

3. Schwartz DH, Dickie E, Pangelinan MM, Leonard G, Perron M, Pike GB, et al. Adiposity is associated with structural properties of the adolescent brain. Neuroimage. 2014;103:192-201.

4. Higuchi S, Kabeya Y, Kato K. Visceral-to-subcutaneous fat ratio is independently related to small and large cerebrovascular lesions even in healthy subjects. Atherosclerosis. 2017;259:41-45.

5. Debette S, Beiser A, Hoffmann U, DeCarli C, O’Donnell CJ, Massaro JM, et al. Visceral fat is associated with lower brain volume in healthy middle-aged adults. Ann Neurol. 2010;68:136-144.

6. Schwartz DH, Leonard G, Perron M, Richer L, Syme C, Veillette S, et al. Visceral fat is associated with lower executive functioning in adolescents. Int J Obes. 2013;37:1336-1343.

7. Syme C, Czajkowski S, Shin J, Abrahamowicz M, Leonard G, Perron M, et al. Glycerophosphocholine Metabolites and Cardiovascular Disease Risk Factors in Adolescents: A Cohort Study. Circulation. 2016;134:1629-1636.

8. Syme C, Pelletier S, Shin J, Abrahamowicz M, Leonard G, Perron M, et al. Visceral fatrelated systemic inflammation and the adolescent brain: a mediating role of circulating glycerophosphocholines. Int J Obes. 2019;43:1223-1230.

9. Marathe GK, Pandit C, Lakshmikanth CL, Chaithra VH, Jacob SP, D'Souza CJM. To hydrolyze or not to hydrolyze: the dilemma of platelet-activating factor acetylhydrolase. $\mathrm{J}$ Lipid Res. 2014;55:1847-1854.

10. Sevastou I, Kaffe E, Mouratis MA, Aidinis V. Lysoglycerophospholipids in chronic inflammatory disorders: The PLA2/LPC and ATX/LPA axes. Biochim Biophys Acta - Mol Cell Biol Lipids. 2013;1831:42-60.

11. Ousman SS, David S. Rapid Recruitment and Activation of Macrophages In the Adult Mouse Spinal Cord. Glia. 2000;104:92-104.

12. Guillemot-Legris O, Muccioli GG. Obesity-Induced Neuroinflammation: Beyond the Hypothalamus. Trends Neurosci. 2017;40:237-253.

13. Chiappelli J, Hong LE, Wijtenburg SA, Du X, Gaston F, Kochunov P, et al. Alterations in frontal white matter neurochemistry and microstructure in schizophrenia: Implications for neuroinflammation. Transl Psychiatry. 2015;5:e548.

14. Hishikawa D, Hashidate T, Shimizu T, Shindou H. Diversity and function of membrane glycerophospholipids generated by the remodeling pathway in mammalian cells. J Lipid Res. 2014;55:799-807.

15. Rong X, Albert CJ, Hong C, Duerr MA, Chamberlain BT, Tarling EJ, et al. LXRs regulate ER stress and inflammation through dynamic modulation of membrane phospholipid composition. Cell Metab. 2013;18:685-697.

16. Pasqualetti G, Brooks DJ, Edison P. The Role of Neuroinflammation in Dementias. Curr Neurol Neurosci Rep. 2015;15.

17. van der Lee SJ, Teunissen CE, Pool R, Shipley MJ, Teumer A, Chouraki V, et al. Circulating metabolites and general cognitive ability and dementia: Evidence from 11 cohort studies. Alzheimer's Dement. 2018;14:707-722.

18. Zhu L, Zhong M, Elder GA, Sano M, Holtzman DM, Gandy S, et al. Phospholipid dysregulation contributes to ApoE4-associated cognitive deficits in Alzheimer's disease pathogenesis. Proc Natl Acad Sci. 2015;112:11965-11970. 
19. Luu W, Zerenturk EJ, Kristiana I, Bucknall MP, Sharpe LJ, Brown AJ. Signaling regulates activity of DHCR24, the final enzyme in cholesterol synthesis. J Lipid Res. 2014;55:410420.

20. Crameri A, Biondi E, Kuehnle K, Lütjohann D, Thelen KM, Perga S, et al. The role of seladin-1/DHCR24 in cholesterol biosynthesis, APP processing and A $\beta$ generation in vivo. EMBO J. 2006;25:432-443.

21. Greeve I, Hermans-Borgmeyer I, Brellinger C, Kasper D, Gomez-Isla T, Behl C, et al. The Human DIMINUTO/DWARF1 Homolog Seladin-1 Confers Resistance to Alzheimer's Disease-Associated Neurodegeneration and Oxidative Stress. J Neurosci. 2000;20:73457352.

22. Lu X, Kambe F, Cao X, Kozaki Y, Kaji T, Ishii T, et al. 3B-Hydroxysteroid- $\Delta 24$ Reductase Is a Hydrogen Peroxide Scavenger, Protecting Cells From Oxidative Stress-Induced Apoptosis. Endocrinology. 2008;149:3267-3273.

23. Wu C, Miloslavskaya I, Demontis S, Maestro R, Galaktionov K. Regulation of cellular response to oncogenic and oxidative stress by Seladin-1. Nature. 2004;432:640-645.

24. Martiskainen H, Paldanius KMA, Natunen T, Takalo M, Marttinen M, Leskelä S, et al. DHCR24 exerts neuroprotection upon inflammation-induced neuronal death. J Neuroinflammation. 2017;14:1-16.

25. Pausova Z, Paus T, Abrahamowicz M, Bernard M, Gaudet D, Leonard G, et al. Cohort Profile: The Saguenay Youth Study (SYS). Int J Epidemiol. 2017;46:e19.

26. Würtz P, Kangas AJ, Soininen P, Lawlor DA, Davey Smith G, Ala-Korpela M. Quantitative Serum Nuclear Magnetic Resonance Metabolomics in Large-Scale Epidemiology: A Primer on -Omic Technologies. Am J Epidemiol. 2017;186:1084-1096.

27. Aulchenko YS, Struchalin M V, van Duijn CM. ProbABEL package for genome-wide association analysis of imputed data. BMC Bioinformatics. 2010;11:134.

28. Kettunen J, Demirkan A, Würtz P, Draisma HHM, Haller T, Rawal R, et al. Genome-wide study for circulating metabolites identifies 62 loci and reveals novel systemic effects of LPA. Nat Commun. 2016;7:11122.

29. Sabourin JA, Brody LC, Wilson JEB, Wilson AF. ComPaSS GWAS : A method to reduce type I error in genome wide association studies when replication data are not available. Genet Epidemiol. 2019:102-111.

30. Lonsdale J, Thomas J, Salvatore M, Phillips R, Lo E, Shad S, et al. The Genotype-Tissue Expression (GTEx) project. Nat Genet. 2013;45:580-585.

31. Trabzuni D, Ryten M, Walker R, Smith C, Imran S, Ramasamy A, et al. Quality control parameters on a large dataset of regionally dissected human control brains for whole genome expression studies. J Neurochem. 2011;119:275-282.

32. Hawrylycz MJ, Lein ES, Guillozet-Bongaarts AL, Shen EH, Ng L, Miller JA, et al. An anatomically comprehensive atlas of the adult human brain transcriptome. Nature. 2012;489:391-399.

33. Mazziotta J, Toga A, Evans A, Fox P, Lancaster J, Zilles K, et al. A probabilistic atlas and reference system for the human brain: International Consortium for Brain Mapping (ICBM). Philos Trans R Soc B Biol Sci. 2001;356:1293-1322.

34. Colantuoni C, Lipska BK, Ye T, Hyde TM, Tao R, Leek JT, et al. Temporal dynamics and genetic control of transcription in the human prefrontal cortex. Nature. 2011;478:519-523.

35. Jaffe AE, Hyde T, Kleinman J, Weinbergern DR, Chenoweth JG, McKay RD, et al. Practical impacts of genomic data 'cleaning' on biological discovery using surrogate variable analysis. BMC Bioinformatics. 2015;16:1-10.

36. Li M, Santpere G, Kawasawa YI, Evgrafov O V., Gulden FO, Pochareddy S, et al. Integrative functional genomic analysis of human brain development and neuropsychiatric risks. Science (80- ). 2018;362:1-13. 
37. Parker N, Vidal-pineiro D, French L, Shin J, Adams HHH, Brodaty H, et al. Corticosteroids and regional variations in thickness of the human cerebral cortex across the lifespan. Cereb Cortex. 2019:1-12.

38. Wang D, Liu S, Warrell J, Won H, Shi X, Navarro FCP, et al. Comprehensive functional genomic resource and integrative model for the human brain. Science (80- ). 2018;362.

39. Lake BB, Ai R, Kaeser GE, Salathia NS, Yung YC, Liu R, et al. Neuronal subtypes and diversity revealed by single-nucleus RNA sequencing of the human brain. Science (80- ). 2016;352:1586-1590.

40. Chen J, Bardes EE, Aronow BJ, Jegga AG. ToppGene Suite for gene list enrichment analysis and candidate gene prioritization. Nucleic Acids Res. 2009;37:305-311.

41. Gaunt TR, Shihab HA, Hemani G, Min JL, Woodward G, Lyttleton O, et al. Systematic identification of genetic influences on methylation across the human life course. Genome Biol. 2016;17:61.

42. Ward LD, Kellis M, LD W, M K. HaploReg: a resource for exploring chromatin states, conservation, and regulatory motif alterations within sets of genetically linked variants. Nucleic Acids Res. 2012;40:D930-4.

43. Goldstein J, Brown M. Regulation of the mevalonate pathway. Nature. 1990;343:425-430.

44. Mahdessian H, Taxiarchis A, Popov S, Silveira A, Franco-Cereceda A, Hamsten A, et al. TM6SF2 is a regulator of liver fat metabolism influencing triglyceride secretion and hepatic lipid droplet content. Proc Natl Acad Sci. 2014;111:8913-8918.

45. Swerdlow DI, Preiss D, Kuchenbaecker KB, Holmes M V., Engmann JEL, Shah T, et al. HMG-coenzyme A reductase inhibition, type 2 diabetes, and bodyweight: Evidence from genetic analysis and randomised trials. Lancet. 2015;385:351-361.

46. Sliz E, Sebert S, Würtz P, Kangas AJ, Soininen P, Lehtimäki T, et al. NAFLD risk alleles in PNPLA3, TM6SF2, GCKR and LYPLAL1 show divergent metabolic effects. Hum Mol Genet. 2018;27:2214-2223.

47. The 1000 Genomes Project Consortium. A global reference for human genetic variation. Nature. 2015;526:68-74.

48. Chong JA, Tapia-Ramirez J, Kim S, Toledo-Aral JJ, Zheng Y, Boutros MC, et al. REST: A mammalian silencer protein that restricts sodium channel gene expression to neurons. Cell. 1995;80:949-957.

49. Lu T, Aron L, Zullo J, Pan Y, Kim H, Chen Y, et al. REST and stress resistance in ageing and Alzheimer's disease. Nature. 2014;507:448-454.

50. Ritchie SC, Würtz P, Nath AP, Abraham G, Havulinna AS, Fearnley LG, et al. The Biomarker GlycA is Associated with Chronic Inflammation and Predicts Long-Term Risk of Severe Infection. Cell Syst. 2015;1:293-301.

51. Kozlitina J, Smagris E, Stender S, Nordestgaard BG, Zhou HH, Tybjærg-Hansen A, et al. Exome-wide association study identifies a TM6SF2 variant that confers susceptibility to nonalcoholic fatty liver disease. Nat Genet. 2014;46:352-356.

52. Luukkonen PK, Zhou Y, Nidhina Haridas PA, Dwivedi OP, Hyötyläinen T, Ali A, et al. Impaired hepatic lipid synthesis from polyunsaturated fatty acids in TM6SF2 E167K variant carriers with NAFLD. J Hepatol. 2017;67:128-136.

53. Sharpe LJ, Wong J, Garner B, Halliday GM, Brown AJ. Is seladin-1 really a selective alzheimer's disease indicator? J Alzheimer's Dis. 2012;30:35-39.

54. Hernández-Jiménez M, Martínez-López D, Gabandé-Rodríguez E, Martín-Segura A, Lizasoain I, Ledesma MD, et al. Seladin-1/DHCR24 is neuroprotective by associating EAAT2 glutamate transporter to lipid rafts in experimental stroke. Stroke. 2016. 2016. https://doi.org/10.1161/STROKEAHA.115.010810.

55. Waterham HR, Koster J, Romeijn GJ, Hennekam RC, Vreken P, Andersson HC, et al. Mutations in the 3b-hydroxysterol Delta24-reductase gene cause desmosterolosis, an 
autosomal recessive disorder of cholesterol biosynthesis. Am J Hum Genet. 2001;69:685694.

56. Andersson HC, Kratz L, Kelley R. Desmosterolosis presenting with multiple congenital anomalies and profound developmental delay. Am J Med Genet. 2002;113:315-319.

57. Prinsen BHCMT, Romijn JA, Bisschop PH, de Barse MMJ, Barrett PHR, Ackermans M, et al. Endogenous cholesterol synthesis is associated with VLDL-2 apoB-100 production in healthy humans. J Lipid Res. 2003;44:1341-1348.

58. Chang Y, Robidoux J. Dyslipidemia management update. Curr Opin Pharmacol. 2017;33:47-55.

59. Cole LK, Vance JE, Vance DE. Phosphatidylcholine biosynthesis and lipoprotein metabolism. Biochim Biophys Acta - Mol Cell Biol Lipids. 2012.

60. Bazan NG. Synaptic lipid signaling: Significance of polyunsaturated fatty acids and plateletactivating factor. J Lipid Res. 2003;44:2221-2233.

61. Jakobsen CH, Størvold GL, Bremseth H, Follestad T, Sand K, Mack M, et al. DHA induces ER stress and growth arrest in human colon cancer cells: Associations with cholesterol and calcium homeostasis. J Lipid Res. 2008;49:2089-2100.

62. Esser D, van Dijk SJ, Oosterink E, Lopez S, Müller M, Afman LA. High fat challenges with different fatty acids affect distinct atherogenic gene expression pathways in immune cells from lean and obese subjects. Mol Nutr Food Res. 2015;59:1563-1572.

63. Braun K, Dhana K, de Vries PS, Voortman T, van Meurs JBJ, Uitterlinden AG, et al. Epigenome-wide association study (EWAS) on lipids: the Rotterdam Study. Clin Epigenetics. 2017;9:1-11.

64. Demerath EW, Guan W, Grove ML, Aslibekyan S, Mendelson M, Zhou YH, et al. Epigenome-wide association study (EWAS) of BMI, BMI change and waist circumference in African American adults identifies multiple replicated loci. Hum Mol Genet. 2015;24:44644479.

65. Morell P, Jurevics H. Origin of cholesterol in myelin. Neurochem Res. 1996;21:463-470.

66. Zhang J, Liu Q. Cholesterol metabolism and homeostasis in the brain. Protein Cell. 2015;6:254-264.

67. Dong W, Guan F, Zhang X, Gao S, Liu N, Chen W, et al. Dhcr24 activates the $\mathrm{PI} 3 \mathrm{~K} / \mathrm{Akt} / \mathrm{HKII}$ pathway and protects against dilated cardiomyopathy in mice. Anim Model Exp Med. 2018;1:40-52.

68. Hatzikotoulas K, Gilly A, Zeggini E. Using population isolates in genetic association studies. Brief Funct Genomics. 2014;13:371-377. 
585

586

587

588

589

590

591

\section{Funding sources}

The Saguenay Youth Study has been funded by the Canadian Institutes of Health Research (TP, ZP), Heart and Stroke Foundation of Canada (ZP), Canadian Foundation for Innovation (ZP) and National Institutes for Health (ZP).

\section{Key words}

white matter, DHCR24, visceral adiposity, phospholipid, lipoprotein, GWAS, adolescence 
Figure legends

Figure 1. The genome-wide significant locus on chromosome 1 near DHCR24. The GWAS of the $1^{\text {st }}$ principal component (PC1) of visceral fat, circulating level of PC(16:0/2:0), and white matter microstructure (T1-weighted signal intensity) was conducted in 872 SYS adolescents using ProbABEL software. The PC1 was calculated using age- and sex-adjusted variables (and height for VF), and the GWAS was further adjusted for differential relationships between participants (unrelated or sibship relationships). The Manhattan plot shows the result of the GWAS (A) and the regional plot shows the association signal on chromosome 1 (B).

\section{Figure 2. Expression of DHCR24 during lifespan.}

Cerebrocortical mRNA expression of DHCR24 was acquired from five post-mortem human databases (Allen Human Brain Atlas, AHBA; BrainCloud; BRAINEAC; BrainSpan; and Genotype-Tissue Expression project; GTEx) with a total of 572 donors. Within each database, mRNA expression was scaled by region and all scaled values were pooled by cortical lobe. Donor age (x-axis) was regressed on lobar expression (y-axis) adjusting for sex as a fixed effect and donor ID as a random effect. Colours indicate the database from which the expression data was obtained.

Figure 3. The effects of the DHCR24 rs588709-G on particle concentration of 14 lipoprotein subclasses and the lipid fractions within the 14 subclasses.

The metabolic effects of the DHCR24 rs588709-G were evaluated in 931 SYS adolescents in linear models, in which metabolite concentrations served as outcomes and DHCR24 rs588709-G allele count $(0,1,2)$ as predictor. Metabolite concentrations were adjusted for age, sex, and family relatedness, and rank transformed to normality. Error bars indicate $95 \%$ confidence intervals. Statistical significance was considered at $p<0.002$ $(0.05 / 29)$ to account for testing of 29 independent metabolic measures.

VLDL, very-low-density lipoprotein; IDL, intermediate-density lipoprotein; LDL, low-density lipoprotein; HDL, high-density lipoprotein; XXL, extremely large; XL, very large; L, large; M, medium; S, small; XS, very small.

Figure 4. The effects of the DHCR24 rs588709-G on lipoprotein particle size, apolipoproteins, cholesterol, triglycerides, phospholipids, amino acids and glycoprotein acetylation.

The metabolic effects of the DHCR24 rs588709-G were evaluated in 931 SYS adolescents in linear models, in which metabolite concentrations served as outcomes and DHCR24 rs588709-G allele count $(0,1,2)$ as predictor. Metabolite concentrations were adjusted for age, sex, and family relatedness, and rank transformed to normality. Error bars indicate $95 \%$ confidence intervals. Statistical significance was considered at $p<0.002$ $(0.05 / 29)$ to account for testing of 29 independent metabolic measures.

VLDL, very-low-density lipoprotein; LDL, low-density lipoprotein; HDL, high-density lipoprotein; ApoB, apolipoprotein B; ApoA-I, apolipoprotein A-I; C, cholesterol; TG, triglyceride; PG, phosphoglyceride; PC, phosphatidycholine; SM, sphingomyelin; GlycA, glycoprotein acetylation.

Figure 5. The overall similarities of the metabolomic effects of DHCR24 rs588709-G versus TM6SF2 rs58542926-T or HMGCR rs12916-T.

The metabolomic effects of the TM6SF2 rs58542926-T and HMGCR rs12916-T were obtained from a publicly available GWAS [28]. The dashed line indicates the linear fit of the effect estimates on 111 metabolic measures that were available in both studies. The shaded area denotes the $95 \%$ confidence interval for the line. $\mathrm{R}^{2}$ indicates the goodness of fit. 
634

635

Table 1. The basic characteristics of the SYS adolescents.

\begin{tabular}{|l|l|l|l|}
\hline & Female & Male & Total \\
\hline $\mathrm{N}$ & 489 & 442 & 931 \\
\hline Age (years) & $15.1 \pm 1.9$ & $15.0 \pm 1.8$ & $15.1 \pm 1.8$ \\
\hline Weight $(\mathrm{kg})$ & $55.9 \pm 12.1$ & $61.6 \pm 16.6$ & $58.6 \pm 14.7$ \\
\hline Height $(\mathrm{cm})$ & $159.8 \pm 6.6$ & $167.3 \pm 10.4$ & $163.4 \pm 9.4$ \\
\hline Body mass index $\left(\mathrm{kg} / \mathrm{m}^{2}\right)$ & $21.8 \pm 4.2$ & $21.8 \pm 4.5$ & $21.8 \pm 4.4$ \\
\hline Visceral fat $\left(\mathrm{cm}^{3}\right)$ & $16.3[11.9-24.4]$ & $13.6[9.6-27.8]$ & $15.5[10.7-25.7]$ \\
\hline White matter T1W-SI (normalized) & $1.20 \pm 0.015$ & $1.20 \pm 0.014$ & $1.20 \pm 0.015$ \\
\hline C-reactive protein $(\mathrm{mg} / \mathrm{l})$ & $0.5[0.2-1.4]$ & $0.3[0.2-0.8]$ & $0.4[0.2-1.1]$ \\
\hline
\end{tabular}

636

637 

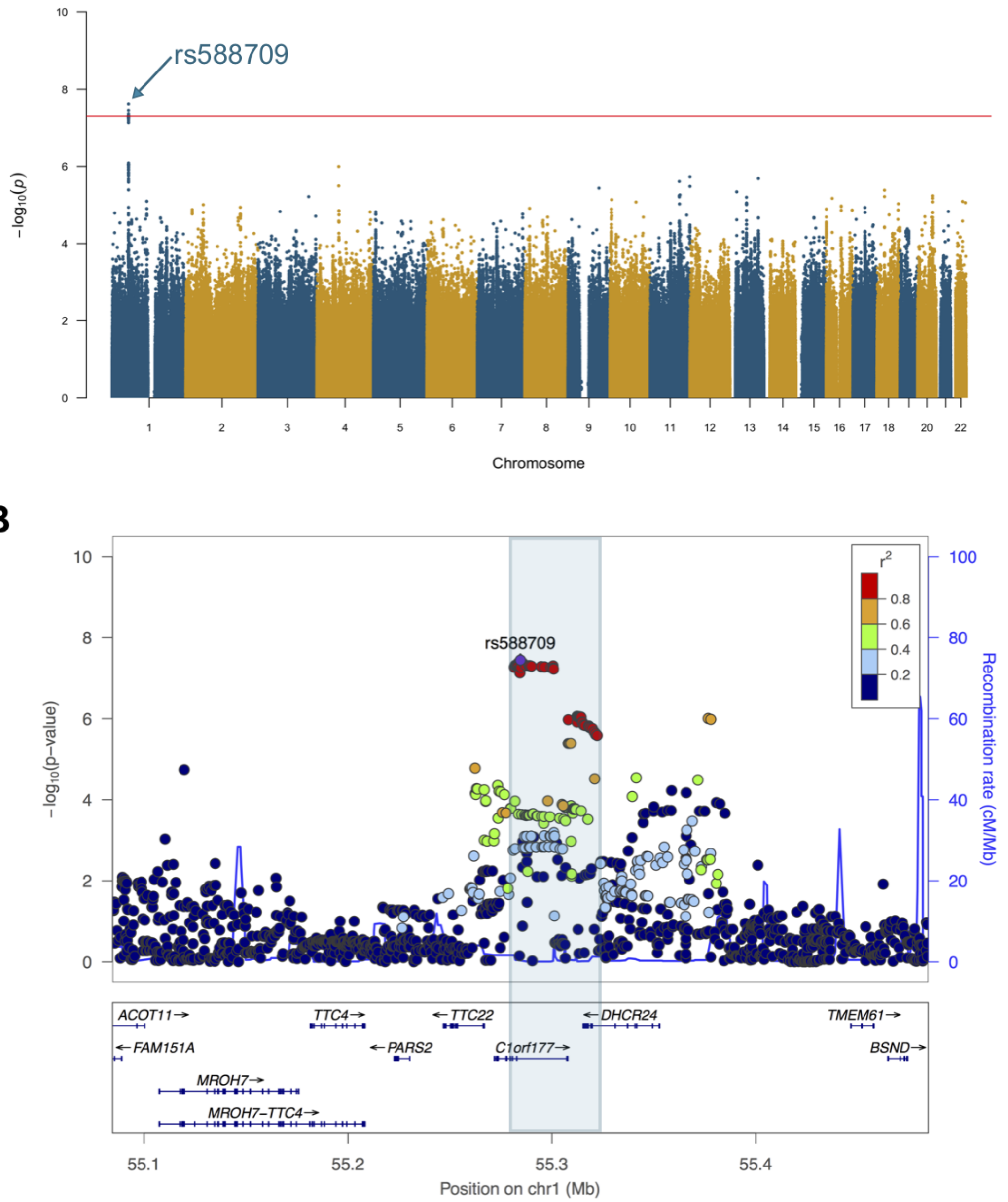


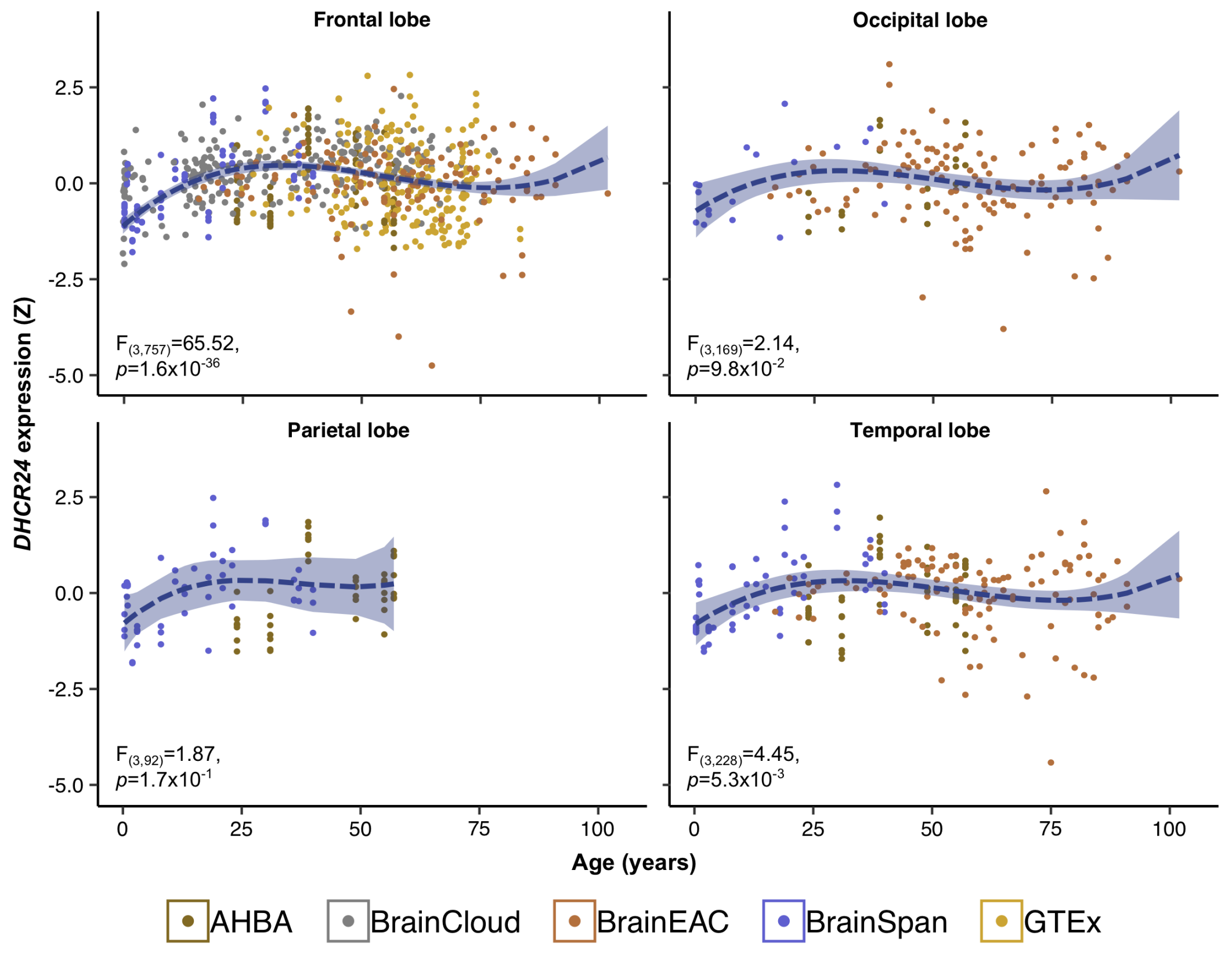




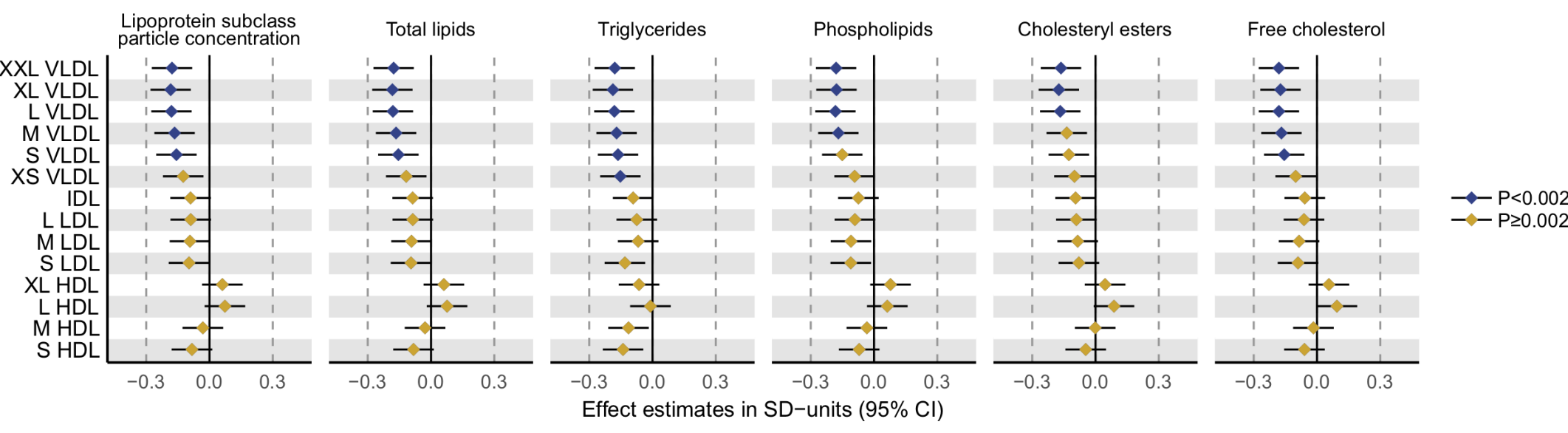




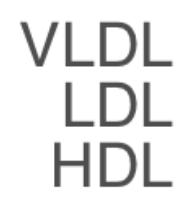

Apolipoproteins

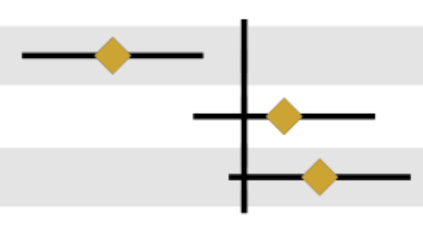

ApoB

ApoB to ApoA1 ratio

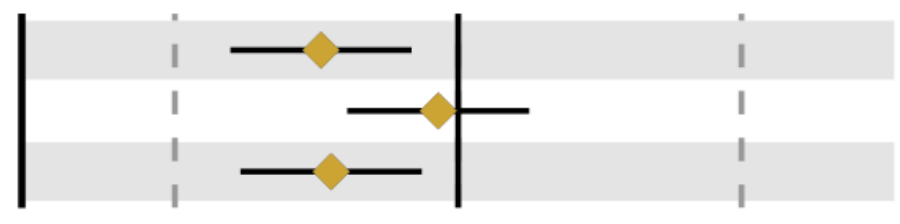

Cholesterol

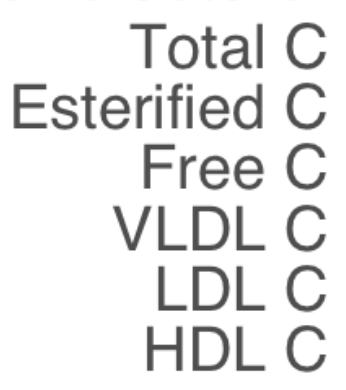

Triglycerides and phospholipids
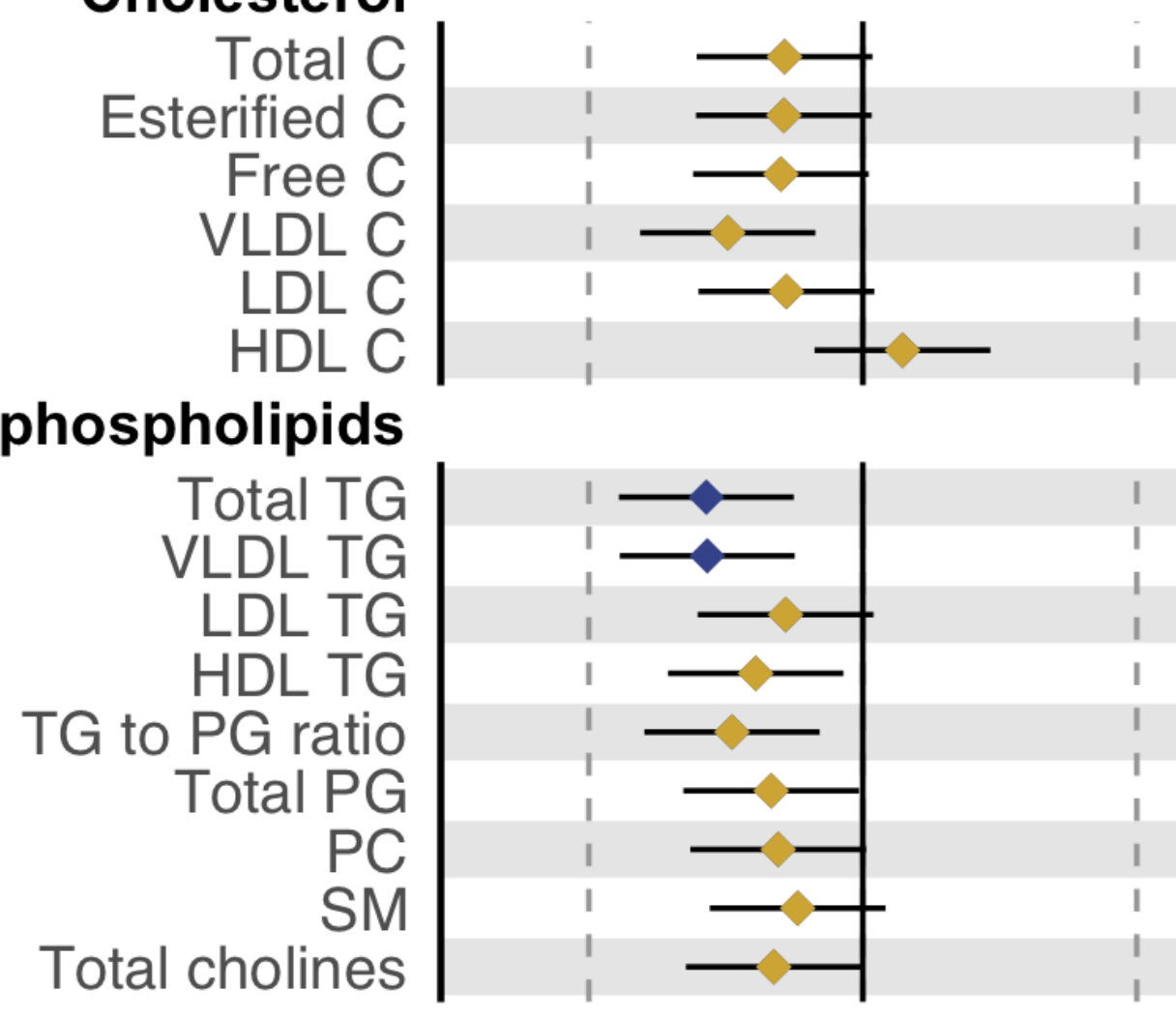

Amino acids

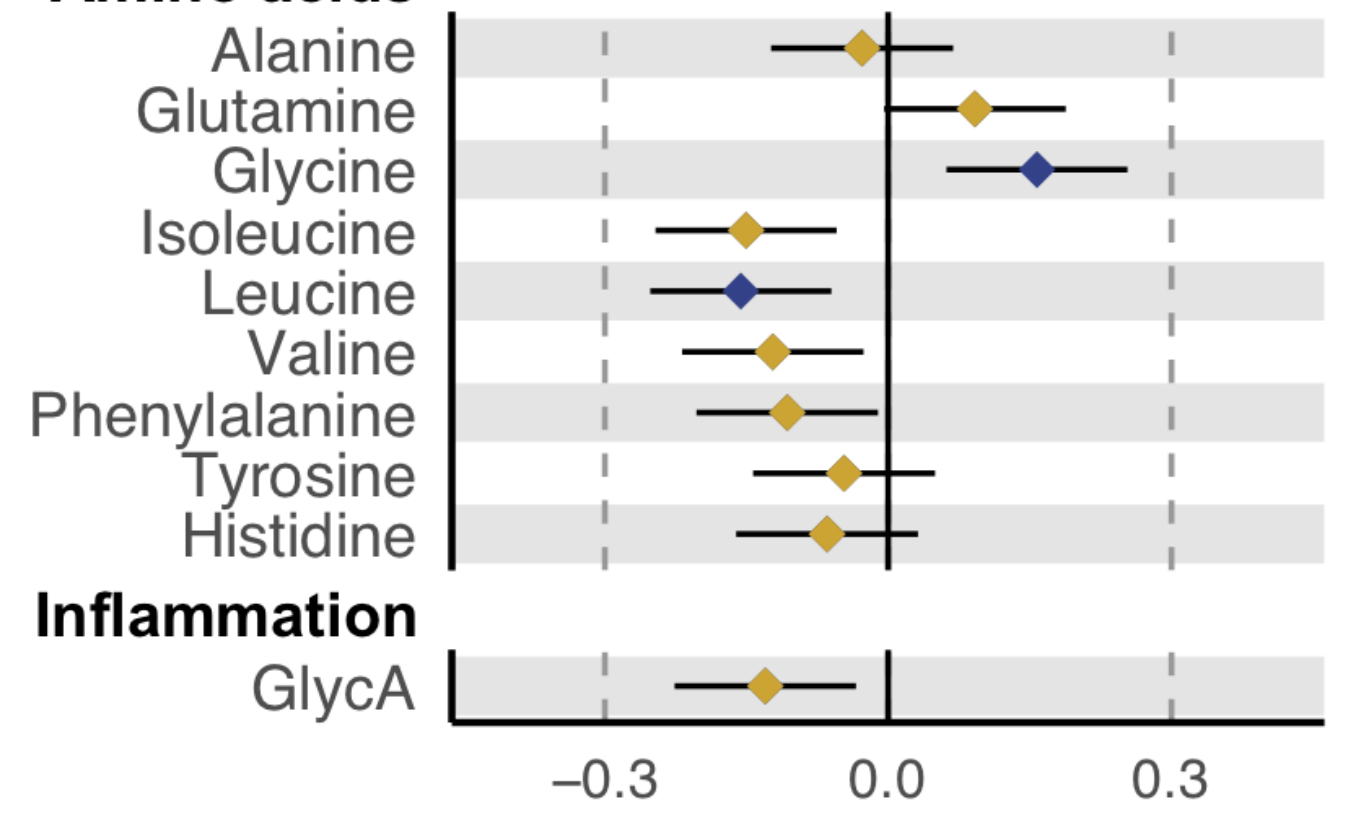

Effect estimates in SD-units $(95 \% \mathrm{Cl})$ 

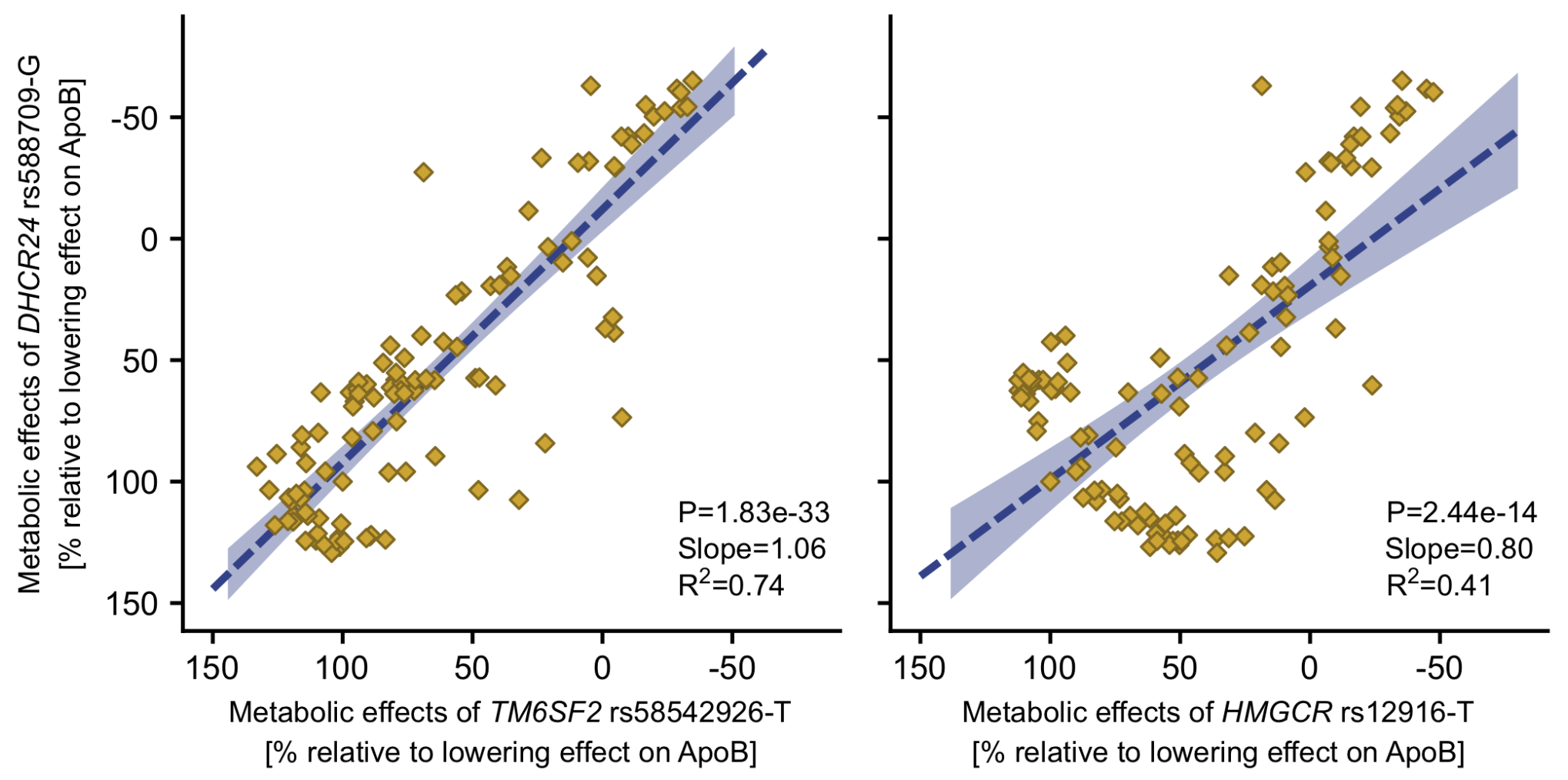